\title{
Les excuses émotionnelles en droit angloaméricain : une illustration de l'intérêt de la philosophie de l'action en droit pénal
}

\section{Fanny-Elisabeth ROLLET}

\section{(2) OpenEdition}

Journals

Édition électronique

URL : https://journals.openedition.org/philonsorbonne/1556

DOI : 10.4000/philonsorbonne. 1556

ISSN : 2270-7336

Éditeur

Publications de la Sorbonne

Édition imprimée

Date de publication : 14 février 2020

Pagination : 77-100

ISSN : 1255-183X

Référence électronique

Fanny-Elisabeth ROLLET, « Les excuses émotionnelles en droit angloaméricain : une illustration de l'intérêt de la philosophie de l'action en droit pénal », Philonsorbonne [En ligne], 14 | 2020, mis en ligne le 20 mars 2020, consulté le 21 septembre 2021. URL : http://journals.openedition.org/ philonsorbonne/1556 ; DOI : https://doi.org/10.4000/philonsorbonne.1556 


\title{
Les excuses émotionnelles en droit angloaméricain : une illustration de l'intérêt de la philosophie de l'action en droit pénal
}

\author{
Fanny-Elisabeth ROLLET
}

Notre contribution se fixe deux objectifs dans le champ de la philosophie du droit pénal comparé. Elle aura premièrement pour objet d'illustrer la structure et la fonction du discours sur l'émotion en droit pénal, en prenant l'exemple de quelques excuses pénales en droit comparé : la contrainte et la provocation, excuses «émotionnelles », c'est-à-dire traditionnellement associées à une émotion - la contrainte associée à la peur, et la provocation associée à la colère. Il s'agira également de montrer par cette étude que le discours majoritaire de la théorie de la responsabilité pénale, fondé sur une théorie mécaniciste de l'émotion et de la responsabilité (dans la lignée du volontarisme de Hart, qui a durablement influencé toute la littérature dans le domaine des excuses) n'est pas susceptible de saisir l'émotion autrement que comme obstacle extérieur à la volonté et comme privation d'agentivité, au lieu de lui conférer un sens véritable. Nous nous tournerons ainsi plutôt vers des théories " cognitives ", ou " évaluatives » de l'émotion ${ }^{1}$, c'est-à-dire vers des approches non mécanicistes, pour voir qu'elles seules permettent, dans le cadre d'une approche de l'action criminelle fondée sur l'agent, d'accomplir une double tâche : rendre tout d'abord possible la saisie d'un sens véritable donné à l'émotion par le droit, et, par là-même, maintenir l'agentivité dans les excuses émotionnelles, par une authentique redescription d'action. On opposera ce maintien d'agentivité à l'incapacité à répondre aux raisons, qui s'illustre dans d'autres types de

1. À la suite, en particulier, de Dan M. Kahan et Martha C. Nussbaum, dans «Two Conceptions of Emotion in Criminal Law ", in Columbia Law Review, Vol. 96, $\mathrm{n}^{\circ} 2$ (Mar., 1996), p. 269-374. 
moyens de défense (I). En matière de défenses, seule une approche favorable à une appréciation qualitative de l'émotion est susceptible de se soustraire à l'écueil d'un «daltonisme » des agents, et de faire apparaître le fondement de certaines excuses majeures du droit pénal (II).

\section{Action, redescription et excuse pénale}

\section{1) Définir l'action, punir l'agent}

Une clarification tout d'abord sur les méthodes et outils qui sont les nôtres, et sur le domaine de notre étude. Nous inscrivons nos analyses dans un cadre conceptuel qui est celui de la philosophie de l'action, en particulier dans sa tradition analytique. Elles sont également circonscrites, quant au champ d'étude, aux schémas intellectuels du droit pénal moderne, dont on résumera les enjeux sous l'impératif de «punir l'agent en tant qu'agent $»^{2}$, articulation de principes structurants où le droit de punir moderne puise à la fois sa légitimité historique et son unité conceptuelle.

Commençons par exposer brièvement les principes renfermés dans l'impératif ci-dessus : " punir l'agent en tant qu'agent » signifie notamment que le droit de punir a été légitimé dès ses commencements philosophiques par une référence nécessaire et suffisante aux qualités essentielles du sujet individuel, adossant ainsi la théorie pénale à tout un volet anthropologique. Cette référence, constante dans le libéralisme politique du $18^{\mathrm{e}}$ siècle, mute ensuite pour devenir, dans la dogmatique juridique du $19^{\mathrm{e}}$ siècle, le programme du droit pénal moderne ${ }^{3}$. Or l'une des façons pour le droit pénal moderne de « coder » l'action humaine, pourrait-on dire, a été de l'enserrer dans une analytique de l'infraction, où cette dernière (crime ou délit) est désormais conçue comme somme d'éléments discrets, devant faire l'objet d'une preuve séparée - ou du moins, indépendants l'un de l'autre dans la doctrine pénale officielle, aux plans conceptuel et probatoire ${ }^{4}$.

2. La formule est, bien sûr, de facture contemporaine : voir par exemple V. Tadros, Criminal Responsibility, Oxford University Press, 2005, p. 100 : «First, an agent is criminally responsible for his action only insofar as that action reflects on him qua agent [...]», mais elle embrasse et décrit toute la tradition du droit pénal moderne (dite aussi «classique » ou « néoclassique » dans la doctrine pénale française), qui porte l'empreinte du libéralisme politique du tournant du $19^{\mathrm{e}}$ siècle. Pour plus de précisions sur ce point, voir notamment : A. Norrie, Law, Ideology and Punishment. Retrieval and Critique of the Liberal Ideal of Criminal Justice, Kluwer Academic Publisher, "Law and Philosophy Library », 1991 ; A. Norrie, Crime, Reason and History, Cambridge University Press, « Law in Context », 2014 ( $3^{\mathrm{e}}$ rééd.), chap. 2 , p. 19-40.

3. Ce volet anthropologique mute par ailleurs en se transformant à l'occasion de son intégration à la doctrine pénale officielle (notamment de common law), mais ce n'est pas là le sujet de notre article. Pour plus de précisions, voir A. Norrie, op. cit., 1991 et op. cit., 2014.

4. Il s'agit bien sûr ici de renvoyer à la combinaison de l'élément moral et de l'élément matériel du délit, qui connaissent ensuite éventuellement des niveaux de subdivision selon les 
Notre proposition initiale est de confronter cet « encodage » de l'action en droit aux catégories de la philosophie de l'action. Nous souhaiterions en particulier interroger le parallélisme, sans doute de façade, entre la physionomie de l'infraction moderne, fondée sur une analytique de l'infraction (mise en place à compter de la seconde moitié du $19^{\mathrm{e}}$ siècle) et, d'autre part, la définition de l'action en philosophie analytique de l'action. Quelle est cette définition? Elle tient essentiellement au fait - si l'on s'appuie par exemple sur la conception davidsonienne, qui nous servira ici de socle conceptuel - que l'action est coextensive à sa rationalité. Sur le fond du camouflage d'une ontologie faite d'événements, l'action ne se détache que par la grammaire à même de la saisir : elle n'est définie que par la capacité pour un événement à pouvoir être décrit en termes intentionnels, c'est-à-dire en termes de "raisons d'agir » de l'agent, ces raisons étant spécifiées en deux sortes d'états mentaux ${ }^{5}$.

La description par les raisons est ainsi constitutive dans l'action, qu'elle soit ou non le fruit d'une description en première personne. Elle s'oppose par exemple à une description du comportement en termes purement causaux, telle que peut l'être le compte-rendu d'un automatisme, d'un mouvementréflexe ou d'un syndrome, qui procèdent à une localisation de la cause inerte du mouvement dans l'organisme de l'individu'. L'agentivité dépend donc intrinsèquement de la possibilité de tenir sur le comportement de l'agent un discours qui se fonde sur des raisons d'agir, celles-ci étant la combinaison de deux composantes, les croyances et les «pro-attitudes $»^{7}$. L'action est ainsi véritablement coextensive à la possibilité de ce discours rationnel sur un cours de conduite : non seulement la description susceptible d'en être faite joue un rôle constitutif dans la qualification - et donc l'application de la sanction pénale, mais la redescription garante d'agentivité peut également servir de marqueur en matière d'autonomie du sujet pénal.

\section{2) La redescription à l'œuvre dans l'excuse}

Comment l'excuse pénale va-t-elle trouver sa place dans le schéma que l'on vient de décrire ? Dans ce cadre conceptuel à la fois davidsonien et

infractions. La doctrine anglo-américaine exprime ces exigences au travers des expressions latines bien connues de mens rea (« intention coupable» ou "volonté de nuire », élément interne de l'infraction) et d'actus reus (« conduite coupable» ou «acte de culpabilité », élément externe de l'infraction). Il ne s'agit pas ici pour nous de contester cette structure officielle de l'infraction, qui subit par ailleurs des assauts dans les divers systèmes de droit, opérant par exemple via des présomptions sectorielles (allègement de la preuve) et des déductions de l'intention à partir de la sophistication de l'élément matériel du crime.

5. Ces raisons doivent aussi être causalement efficaces pour Davidson, mais il paraît inutile d'aborder ce point ici dans le cadre des excuses émotionnelles.

6. Cette distinction prendra tout son sens en I) 2).

7. Tandis que les croyances visent la correspondance de leur contenu propositionnel au monde, les pro-attitudes, comme le désir, visent à faire advenir dans le monde leur contenu propositionnel d'état mental. Ces concepts sont considérés comme étant intrinsèquement normatifs. 
anscombien $^{8}$, où le terme anscombien de description accompagne comme on l'a vu cette définition de l'action "en termes de langage », l'excuse s'interprète comme une "redescription" de l'action, intervenant après la description de l'action par l'accusation. Or cette notion joue également un rôle tout à fait central dans l'excuse. Elle permet en effet de faire le départ entre le domaine de l'agentivité, recouvrant les excuses que l'on désignera comme « intelligibles », et celui des moyens de défense ne postulant pas la rationalité du comportement, voire hypothéquant fortement cette possibilité d'explication rationnelle. La première catégorie d'excuses est susceptible d'une reformulation en termes de raisons et préserve la compétence rationnelle de l'agent: elle postule donc, à un certain degré au moins, la rationalité du comportement décrit ; dans la seconde catégorie, au contraire, celle des défenses "non rationnelles »", la vertu exonératoire de l'excuse ne dépend pas de la fourniture de raisons (de croire ou d'agir), mais de la fourniture d'une expertise, par exemple, ou, plus généralement, d'explications mécanicistes du comportement.

Une précision terminologique s'impose, au vu de notre corpus de référence - le droit pénal angloaméricain constituant le pivot de l'analyse, bien qu'elle puisse s'appliquer à d'autres systèmes de droit. Le terme de "défense» employé ici constitue la traduction très imparfaite du terme anglais defense/defence. Il revêt en langue anglaise juridique le sens de «moyen de défense », typifié et explicite dans le droit, fourni par l'agent et lui servant à modifier les charges qui pèsent contre lui par une redescription de son acte. Ces defenses recouvrent trois catégories que nous identifions comme étant les justifications, les excuses (authentiques) et les exemptions (ou « excuses inauthentiques »). Ces trois catégories distinctes expriment en outre des rapports sensiblement différents à l'agentivité : les exemptions font disparaître l'agent (bien que les doctrines et approches de l'insanity soient multiples, elles semblent annuler toute possibilité de description intelligible, et donc toute agentivité), les justifications (dont le statut d'action ne pose pas

8. Voir notamment Donald Davidson, «Actions, Reasons and Causes », in The Journal of Philosophy, Vol. 60, $\mathrm{n}^{\circ}$ 23, American Philosophical Association, Eastern Division, Sixtieth Annual Meeting (Nov. 7, 1963), p. 685-700 ; G.E.M. Anscombe, Intention, Basil Blackwell, 1957 ( $2^{\text {nd }}$ éd., Harvard University Press) ; et G.E.M. Anscombe, "Under a Description », in Noûs, Wiley, Vol. 13, n 2 (May, 1979), p. 219-233. Les différences que l'on peut relever entre ces deux présentations de l'agent et de l'action seront considérées comme mineures au vu du sujet de cet article, et sont de toute façon immatérielles à notre sens concernant la question de l'excuse - bien qu'elles possèdent un autre relief lorsqu'on aborde d'autres matières, concernant par exemple le traitement de la négligence par le droit pénal.

9. Ne traitant pas spécifiquement de cette seconde catégorie (les excuses non intelligibles, ou « inauthentiques » selon notre caractérisation) dans le cadre de cet article, nous nous contenterons de relever qu'une distinction est possible conceptuellement entre les défenses «non rationnelles » et le constat d' «irrationnalité », qui, comme l'a relevé D. Davidson, condamne le récit sur l'action à des «paradoxes». Voir D. Davidson, "Paradoxes of irrationality », in Problems of Rationality, Clarendon Press, 2004, p. 169 : " The idea of an irrational action, belief, intention, inference, or emotion is paradoxical. For the irrational is not merely the non-rational, which lies outside the ambit of the rational ; irrationality is a failure within the house of reason $»$. 
problème, comme pour la légitime défense et la défense de nécessité), et enfin les excuses véritables, qui demeurent sous la modalité de l'action, et coïncident avec une redescription authentique du comportement. C'est de cette seule catégorie des excuses véritables, centrale dans le tableau fourni ${ }^{10}$, qu'il sera question ici.

Comme tout moyen de défense, la défense de provocation (y compris sous ses figures modernes) engage activement le sujet pénal dans une redescription de son action $^{11}$, redescription qui s'opère à la lumière de raisons «supplétives », à la fois auxiliaires et nouvelles, venant en renfort pour " défaire » une première description de l'action faite par l'accusation, afin d'en modifier le sens et la portée juridique. Le plus souvent, cette redescription va dilater ou rétracter la séquence narrative de l'action intentionnelle dans le temps et l'espace, en procédant à l'ajout de circonstances, de conséquences et de buts, en fournissant de manière générale une nouvelle téléologie, non visible de prime abord, et à l'œuvre dans sa conduite. Le mode opératoire de l'excuse rend donc sensible le problème du séquençage de l'action, qui permet de la requalifier, c'est-à-dire de la faire passer d'une catégorie juridique à une autre. Toutefois, ces raisons d'agir sont complètes concernant la justification - ce sont effectivement des raisons d'agir, qui justifient le cours de conduite dans son intégralité (de sa motivation à son résultat), mais sont pour ainsi dire « tronquées » dans le cas de l'excuse, en ce sens qu'elles ne portent que sur les croyances et sur la motivation de l'agent : ce ne sont, à proprement parler, que des raisons de croire, d'éprouver et de ressentir qui sont alors fournies dans le cadre de l'excuse émotionnelle.

La distinction cardinale entre redescriptions d'action authentiques et non authentiques (qui ne permettent pas, au contraire des premières, de maintenir une agentivité véritable) peut être illustrée par une opposition entre deux types de moyens de défense récurrents dans la jurisprudence angloaméricaine. Il s'agit, d'une part, de la diminished responsibility, forme d'anomalie ou de déficience mentale garantissant une forte réduction de peine à l'accusé d'un meurtre - crime sanctionné, en droits anglais et américain, d'une peine de perpétuité obligatoire. La diminished responsibility joue un rôle subsidiaire et prend le relai des doctrines de l'insanity en raison (notamment) du caractère fréquemment sous-inclusif de ces dernières. Elle penche pour cette raison plutôt vers les catégories d'exemption. L'excuse de provocation, d'autre part, et ses avatars modernes post-réforme tels que l'extreme emotional disturbance (EED) en droit américain $^{12}$, produit également un effet d'exonération partielle pour les

10. Voir tableau en fin de partie I, p. 86.

11. Voir J. L. Austin, «A Plea for Excuses », in Proceedings of the Aristotelian Society, « New Series », Vol. 57, 1956-1957, p. 2 : «In each case the defence, very soundly, insists on a fuller description of the event in its context », discutant de la parenté du mode opératoire des défenses de justification et d'excuse au plan du langage ordinaire.

12. Voir Model penal code, $\S 210.3$.(1)(b) : « (1) Criminal homicide constitutes manslaughter when : [...] (b) a homicide which would otherwise be murder is committed under the 
auteurs de meurtres, mais ses conditions en font une excuse intelligible, reposant sur une authentique redescription d'action. Or, l'usage parfois concurrent qui a pu être fait de ces deux moyens de défense devant les cours prête à confusion, confusion que l'on peut à son tour illustrer par une célèbre affaire américaine. Si l'on repart des faits bien connus du cas Judy Norman, il faut rappeler qu'il s'agit d'une femme qui, ayant tué son mari un aprèsmidi pendant qu'il faisait la sieste, suite à de très nombreuses années d'abus physiques et moraux, a pu envisager diverses lignes de défense. Elle a tout d'abord cherché à établir que, suite à ces abus prolongés (son mari ne travaillant pas et la soumettant entre autres à une prostitution régulière), elle avait subi des menaces récentes, également dirigées contre sa famille proche, et était convaincue que son mari finirait bientôt par les mettre à exécution. Par ailleurs, il est établi qu'elle ne pouvait lui échapper autrement et trouver un réel refuge ailleurs. La légitime défense lui étant de toute façon inaccessible dans sa définition traditionnelle, faute de menace imminente ${ }^{13}$, elle a pu vouloir se tourner vers la provocation : mais l'interprétation de cette défense devant les cours a traditionnellement favorisé les réactions soudaines, " explosives », et non pas une accumulation de provocations dans le temps, ne laissant ainsi pas place à une vision de l'émotion exonératrice comme «combustion lente »[slow burn $]^{14}$, ce qui a depuis fait l'objet de critiques, venues dans un premier temps de la théorie féministe du droit pénal $^{15}$. Ne reste plus alors à ce type d'accusée, pour échapper à la peine obligatoire de perpétuité pour meurtre, qu'à se tourner vers une défense de « responsabilité atténuée » [diminished responsibility], établie dans son cas à

influence of extreme mental or emotional disturbance for which there is a reasonable explanation or excuse. The reasonableness of such explanation or excuse shall be determined from the viewpoint of a person in the actor's situation under the circumstances as he believes them to be ».

13. Voir les décisions correspondantes : Norman v. State, 366 S.E.2d 586 (N.C. App. 1988), où la cour d'appel critique l'erreur de la juridiction de première instance (trial court) de n'avoir pas présenté dans les instructions au jury la possibilité de délibérer sur la légitime défense, ce qui aurait pu conduire à l'acquittement de l'accusée; Norman v. State, 378 S.E.2d 8 (N.C. 1989), et en particulier les $\S \S 35-41$, où les instructions au jury en matière de légitime défense sont refusées, c'est-à-dire où le juge (la cour suprême de Caroline du Nord) décide que ce moyen de défense ne peut s'appliquer aux faits jugés.

14. Jonathan Herring, Criminal Law. Texts, Cases and Materials, Oxford University Press, $7^{\mathrm{e}}$ éd., 2016, p. 369. Voir à ce sujet, en droit anglais, pour des faits similaires, $R$. v. Ahluwalia: "The passage of time following the provocation may also show that the subsequent attack was planned or based on motives, such as revenge or punishment, inconsistent with the loss of self-control and therefore with the defence of provocation " (R. v. Ahluwalia, [1992] 4 All ER 889 (CA), [1993] 96 Cr App Rep 133 ; [1993] Crim LR 63, CA ; [1992] 142 NLJ 1159.

15. Ces nombreuses voix critiques (parmi lesquelles celles de A. Coughlin, M. Baron, $\mathrm{V}$. Nourse...) pointaient le fait qu'interpréter la colère comme explosive revient à renforcer et encourager les traits typiques voire stéréotypiques de l'identité masculine. Sous la neutralité de la référence au self-control (et sous l'universalité, plus ancienne, du discours de concession à la «nature humaine») se nicherait en fait une indulgence marquée envers une certaine catégorie de justiciables (ici, les hommes en colère, se disant provoqués par une infidélité ou un affront). 
l'aide d'un syndrome nommé le Battered Women Syndrome ${ }^{16}$. Ce syndrome est par ailleurs plus facile à établir, dans la mesure où les actes et la motivation de l'auteur ne font plus l'objet d'une évaluation sur le fond d'une norme de conduite - en particulier celle de l'agent raisonnable [reasonable person]. Judy Norman fut finalement condamnée à une peine de six ans d'emprisonnement.

Toutefois, ce choix de défense par défaut n'est pas sans conséquence. Comme l'explique John Gardner dans Offences and Defences ${ }^{17}$, les deux moyens de défense - provocation d'une part, responsabilité diminuée de l'autre - opèrent de manière totalement disjointe. Sous réserve que l'on adopte une théorie adéquate de l'émotion, la défense de provocation maintient une prétention à la rationalité, en représentant l'émotion sur le fondement de laquelle l'auteur a agi comme étant elle-même fondée ou « raisonnable »: on présentera ainsi sa colère comme défendable dans l'excuse de provocation, ou sa peur comme raisonnable eu égard aux pressions subies par l'agent dans l'excuse de contrainte, bien que l'acte entier - un homicide, pour l'acte "provoqué », et tout crime et délit excepté le meurtre, pour l'acte effectué sous la contrainte - ne le soit pas. Le syndrome, quant à lui - voire même la « syndromisation » rampante de cette catégorie d'accusées (dont la conduite subit une réduction systématique au syndrome $)^{18}$, ne permet pas d'établir une redescription authentique de l'action : c'est, au mieux, une manière de faire l'économie de la question de l'action en écartant celle de la rationalité de la conduite, dans la mesure où la réaction est purement causée par une anormalité mentale, et non pas éclairée à partir de raisons. La cause de la réaction homicide est ainsi localisée dans la matérialité inerte de l'organisme, telle qu'altérée par le traumatisme ${ }^{19}$. C'est pourquoi cette vision de l'excuse, dénuée de tout souci d'intelligibilité de la conduite, est de part en part « mécaniciste $»^{20}$ : dès lors que le préjudice se trouve imputé à un état pathologique de «l'agent», ne reste plus de l'émotion que sa force perturbatrice. Seule fait foi l'intensité de cet état de privation de discernement, l'action demeurant irrémédiablement inintelligible, et donc perdue ${ }^{21}$.

16. L'expertise en la matière fut admise suite à State v. Kelly, 97 N. J. 178 ; 478 A2d 364 (1984).

17. Voir en particulier « Justification and Reasons » (ch. 5) ; « The Gist of Excuses » (ch. 6) ; «Provocation and pluralism»(ch. 8), in Offences and Defences : Selected Essays in the Philosophy of Criminal Law, Oxford University Press, 2007.

18. J. Loveless, «R. v. GAC : Battered Woman "Syndromization" », in Criminal Law Review, 2014, p. 655.

19. J. Gardner, « The Gist of Excuses », in op. cit., p. 133-134. La seule réponse possible à un tel comportement, commente l'auteur, est de dire que ce dernier «n'a aucun sens » et doit être mis sur le compte d'un état pathologique.

20. Selon l'opposition très fructueuse dégagée par M. Nussbaum et D. M. Kahan dans leur article, op. cit., p. 6 sq.

21. En un sens, cette solution - correcte au plan de la responsabilité conséquentielle, car elle réduit effectivement la peine prononcée - est la pire qui soit au plan de la responsabilité en un 
Enfin, et en guise de clôture de cette présentation des outils qui sont les nôtres, on peut tenter une synthèse des traits de l'excuse véritable. Pour être une redescription d'action authentique, en effet, l'excuse suppose (au moins) deux éléments : premièrement, il est nécessaire que l'agent réaffirme dans et par le récit de l'excuse sa compétence rationnelle, et qu'il ne soit pas automatiquement destitué comme être rationnel par le simple fait d'être excusé. Gardner dira même que ces êtres « rationnellement destitués » n'ont justement «plus besoin d'excuses ». Deuxièmement, et c'est là une nuance importante, il semble que l'excuse ne milite pas directement en faveur de l'action commise (comme le ferait la justification directe de l'action, et du cours intégral de cette action - ce qui a lieu par exemple dans la légitime défense), mais qu'elle opère en pointant vers des circonstances de l'action qui, elles, militent en faveur des croyances et émotions sur le fondement desquelles l'agent a agi ${ }^{22}$. L'excuse opère donc en pointant le caractère approprié de l'émotion, appuyée sur des états cognitifs de l'agent : lui ôter ce caractère de justification «partielle» ou « indirecte» reviendrait à la confondre avec une explication purement causale du comportement, et donc un déni de toute responsabilité. Battant en brèche cette confusion ou ce chevauchement illégitime, John Gardner explique encore pourquoi l'excuse dérive en réalité de la justification, et peut être conçue comme justification indirecte ou imparfaite ${ }^{23}$ :

The structure of excuse derives [...] from the structure of justification, and thus shares in its combination of subjective (explanatory) and objective (guiding) rationality ${ }^{24}$.

True, excused actions are unjustified ones. Nevertheless, in making an excuse one relies on the fact that one's unjustified action was taken on the strength of a justified belief or attitude or emotion, etc. An excuse for an angry action, qua angry, depends on the justification of the anger itself. Even in the realm of excuse, therefore, the analysis is moral and not merely causal, for the question of the justification of the anger (and hence the excuse of the angry action) is a moral question. Those who take the opposite view fall into the trap of confusing excuses with denials of responsibility ${ }^{25}$.

sens plus «fondamental » dégagé par Gardner, c'est-à-dire comme capacité à donner une explication de sa conduite par des raisons (voir notamment "The Mark of Responsibility », in op. cit., p. 180-182). L'émotion purement incapacitante, qui participe d'une «fausse redescription », ne saurait en effet ressortir à de « bonnes raisons » d'agir.

22. J. Gardner, «In Defence of Defences », in op. cit., p. 86. Ces circonstances de l'action ne sont pas nécessairement formulées en termes subjectifs.

23. " One of the biggest sources of confusion in the study of excuses lies in the thought that those who make excuses are thereby denying their responsibility for their actions » (John Gardner, «The Gist of Excuses », in op. cit., p. 131).

24. J. Gardner, « Justifications and Reasons », in op. cit., p. 109.

25. J. Gardner, « Provocation and Pluralism », in op. cit., p. 160-161. 
Cette distinction entre raisons d'agir « complètes » (dans la justification) et raisons de croire ou d'éprouver (dans l'excuse) apparaît, de loin en loin, dans la doctrine et la jurisprudence angloaméricaine - notamment devant les juridictions du degré le plus élevé. Elle figure par exemple dans les termes d'une décision récente sur la provocation, rendue par la Cour suprême de Californie ${ }^{26}$, qui martèle dans les instructions au jury la distinction devant être faite entre "caractère raisonnable de l'acte» commis [act reasonableness], non nécessaire à la défense - autrement, l'acte serait bien sûr justifié et il ne s'agirait plus d'excuser l'accusé - et, d'autre part, le « caractère raisonnable de l'émotion » [emotion reasonableness], qui, en revanche, doit nécessairement être établi pour faire valoir une telle excuse. L'excuse émotionnelle revêtirait ainsi le statut d'une "explication rationnelle indirecte $»^{27}$, porteuse d'une exigence de rationalité de l'émotion, mais non de l'acte dans son entier.

En dépit de ces distinctions, il semblerait que le traitement de l'impulsivité en droit pénal ait en grande partie négligé cette donnée fondamentale de l'explication sous-jacente à l'excuse émotionnelle, en la traitant de manière presque automatique comme l'envers pur et simple de l'action ${ }^{28}$. Il s'agira donc, dans les lignes qui vont suivre, d'interroger cette référence à l'émotion en droit pénal sans tomber dans le travers classique de son assimilation automatique à une cause de privation d'agentivité - c'està-dire sans se laisser aller à cette vision paresseuse, finalement assez routinière, de l'excuse.

26. Voir People v. Beltran, 301 P. 3d 1120 (Cal. 2013). Sur cette décision : «The defense asserted that the judge erred by implying that the defendant's act of killing had to be reasonable and that adequate provocation exists simply when a reasonable person would have been provoked to act rashly (sometimes called "emotion reasonableness") », Aya Bruber, « A Provocative Defense », in California Law Review, vol. 103, n² 2, 2015, p. 273 ; p. 275-276.

27. Pour l'intégration de ces catégories à une théorie générale des moyens de défense, voir notre tableau en fin de partie I, p. 86.

28. Cette conception paresseuse de l'excuse comme incapacité à répondre aux raisons est selon nous en partie due aux ambiguïtés d'un passage de l'article fondateur d'Austin sur les excuses, brèche dans laquelle toute la lignée "hartienne" de la théorie du droit a pu s'engouffrer, et qui pèse encore sur les classifications actuelles des defences : opposant justification et excuse dans le passage précédent, Austin écrit en effet que « To take this line is to justify the action, to give reasons for doing it ; not to say, to brazen it out, to glory in it, or the like. A different way of going about it is to admit that it wasn't a good thing to have done, but to argue that it is not quite fair or correct to say baldly "X did A" " (J. Austin, "A Plea for Excuses », in Proceedings of the Aristotelian Society, « New Series », Vol. 57, 19561957, p. 3). 
TABLEAU DE SYNTHÈSE DES DEFENCES EN DROIT DE COMMON LAW

(Incidence sur la redescription d'action, la qualification et l'imputation)

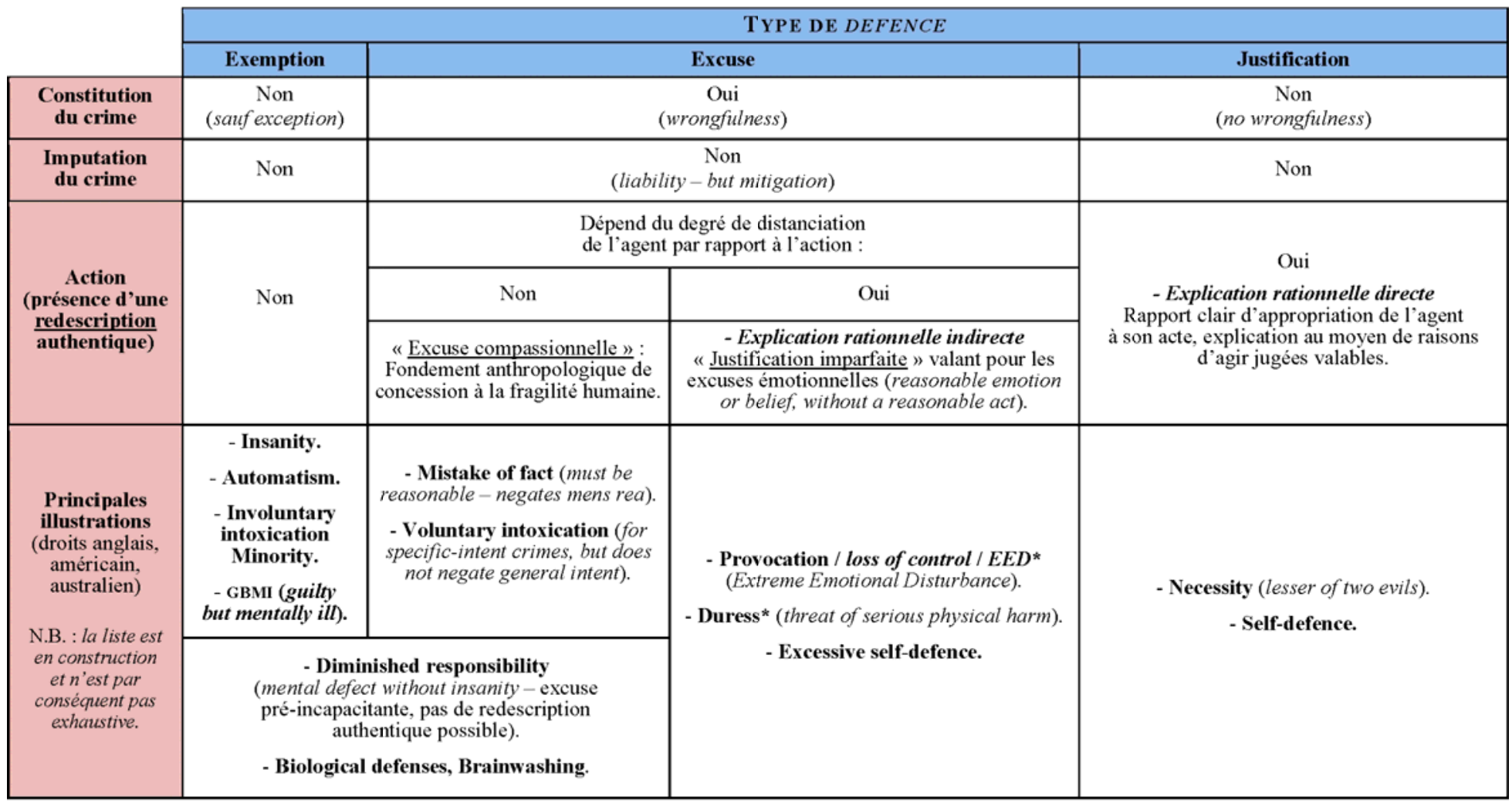




\section{Le problème de l'agentivité dans les excuses émotionnelles}

On se penchera à présent sur le cas emblématique des excuses émotionnelles, désignant sous ce vocable les excuses de contrainte et de provocation en droit de common law. Associant traditionnellement ces excuses, dans leur définition et leur fondement, à une émotion particulière - respectivement la peur et la colère ${ }^{29}$, le langage du droit est souvent pléthorique pour s'y référer ${ }^{30}:$ par exemple, la langue juridique post-réforme parlera plutôt de «loss of control» (dans sa version anglaise) ou de « désordre émotionnel extrême» (dans sa version américaine issue du MPC - Model Penal Code -, en vigueur dans certains États américains) que de " heat of passion », appellation ancienne de la défense.

L'objectif de ce second temps de l'analyse sera de montrer que l'évaluation de l'émotion comme « appropriée » ou « inappropriée » dans la situation de l'agent, et non sa seule identification comme quantum de force perturbatrice, s'inscrivant au-delà d'un certain seuil, est indispensable pour faire apparaitre le fondement de ces excuses majeures. La théorie évaluative des émotions joue un rôle crucial dans l'explication des distinctions de traitement entre les agents dans le domaine du voluntary manslaughter (qui concerne à la fois la provocation et la contrainte). Nous limiterons les exemples à l'excuse de provocation dans les limites de cette étude, mais l'analyse qui suit serait parfaitement applicable à la contrainte, étant donné le parallélisme structurel unissant les deux moyens de défense. Gardant à l'esprit les exigences de la redescription authentique, nous tâcherons d'établir que seule l'évaluation qualitative de l'émotion permet de conserver à l'excuse toute son intelligibilité. On entend ainsi éviter un double écueil : on se soustrait, d'une part, à une vision de l'excuse comme pure aberration théorique, «anomalie » dans un paradigme général de responsabilité pénale individuelle; d'autre part, on se tire de l'ornière que constitue l'assimilation abusive de l'excuse à de pures hypothèses de responsabilité diminuée, voire aux cas d'incapacité (tels que l'abolition du discernement ou la diminished capacity) qui en constituent la polarité extrême, logiquement situé aux marges de l'action.

On donnera un aperçu des avantages de cette approche «non paresseuse » de l'excuse par la théorie évaluative de l'émotion (2), après avoir rappelé les traits pertinents des excuses émotionnelles (1). On présentera enfin sous forme d'hypothèse de travail succincte l'un des apports

29. Ces excuses émotionnelles abritent, sous la figure juridique de la provocation, le « crime of passion ", objet de culture à la fois populaire et pénale qui a récemment suscité l'intérêt du législateur anglais, américain ou australien par exemple. Le crime de passion est utilisé pour décrire le meurtre d'un proche quand le motif d'un tel meurtre est compris, intelligible pour le public, bien que son acte ne soit pas entièrement défendable et reste réprimé.

30. Pour les appellations concurrentes de l'excuse de provocation, on renverra au tableau en fin de partie I, p. 86 . 
majeurs de cette approche évaluative de l'émotion en droit, à savoir qu'elle permet plus naturellement de faire le lien avec le contexte social et politique de l'excuse (3).

\section{1) Éléments de contexte sur les excuses émotionnelles}

Les éléments de définition des différentes excuses émotionnelles affichent une structure très similaire en droit angloaméricain ${ }^{31}$. Si l'on retient comme définition matricielle leur reformulation récente en droit anglais, ces moyens de défense connaissent trois grandes composantes. Ils sont constitués, d'une part, de deux éléments narratifs, correspondant à une double causalité, et, d'autre part, d'un élément objectif ou «test» visant à évaluer in fine le caractère «raisonnable» de la «réaction». Dans le cas de la provocation (ou loss of control, dans sa formulation la plus récente), la "réaction» en question est nécessairement un homicide. Concernant les éléments narratifs de la défense de provocation, il faut pouvoir établir 1) que l'acte homicide résulte d'une perte de sang-froid ${ }^{32}$, et 2) que cet état émotionnel caractéristique était causé en amont par un élément déclencheur valide, cause éligible à même de justifier pleinement cette émotion. Notons cependant que, contrairement aux exigences valant pour la légitime défense des personnes, l'auteur des faits (tel le mari jaloux qui tue sa femme, cas archétypique de provocation prévu dans la version originelle de la défense) n'a pas à prouver que la victime avait commis un acte illégal afin de fonder sa défense partielle pour homicide. Le troisième élément, généralement regardé comme " objectif», est un élément factuel et donc laissé à l'appréciation du jury : le caractère raisonnable de la réaction est mesuré par sa conformité au standard de la personne raisonnable du même âge et du même sexe que l'accusé. Dans le cas de la contrainte, la double causalité décrite ci-dessus et le standard de conduite opèrent d'une manière structurellement proche: l'auteur du délit ou crime (homicide exclu) doit agir sous une menace considérée comme suffisante (threat of death or grievous bodily harm - c'est l'élément déclencheur valide) ayant provoqué la peur d'une atteinte grave et imminente pour lui-même ou pour un proche. Et de même, son comportement ne doit pas s'écarter d'un standard de reasonable firmness, de résistance de l'homme ordinaire.

Ces excuses très anciennes se présentent comme des récits typifiés sur l'action. Ces derniers sont une illustration parfaite de la tension entre les conceptions anthropologiques et morales sous-jacentes au droit pénal d'une

\footnotetext{
31. Bien que l'on ne s'intéresse pas ici principalement au droit français, qui ne comporte plus de défense de provocation depuis 1994, on souligne que le fait justificatif de contrainte connaît sans doute une définition plus large et bien moins structurée, ce qui ne garantit nullement - loin de là - une application plus favorable au justiciable.

32. Il y aurait beaucoup à dire sur le vocabulaire lié à la perte de contrôle qui est la signature de la formulation moderne de la défense. On renvoie ici à l'étude stimulante d'Alan Norrie sur cette question (Crime, Reason and History. A Critical Introduction to Criminal Law, Cambridge University Press, « Law in Context», $3^{\mathrm{e}}$ éd., 2014, chap. 8 et 11).
} 
part, et le formalisme abstrait, dominant dans le droit pénal moderne, de ses catégories en matière de responsabilité et d'exonération d'autre part. Dans le droit de la provocation, la référence au «loss of control» (et, auparavant, à la « nature humaine ») viennent précisément cristalliser cette opposition.

Laissés à l'appréciation du jury ${ }^{33}$, ces moyens de défense permettent en effet de faire ressortir une certaine dualité dans le traitement de l'action (et de l'agent, au travers de la personne raisonnable) en droit pénal. D'un côté, en effet, le discours juridique (et, souvent, culturel) sur les excuses émotionnelles est marqué par une rhétorique anthropologique négative et universalisante dans sa formulation, rhétorique de concession à la « faiblesse humaine». Suivant un stéréotype de la «passion ingouvernable», la référence à la passion opère comme un appel à la compassion pour l'auteur de l'action, en raison du caractère insurmontable de l'émotion éprouvée. Le discours séculaire et éculé sur le "crime de passion» en serait ici une illustration exemplaire ${ }^{34}$. Ce type de rhétorique, à la fois floue et universalisante, fait dégénérer l'agent en une sorte de sub-agent, ne proposant de son acte qu'une seule lecture: le crime comme réponse moralement involontaire au danger (dans la contrainte au sens traditionnel) ou à l'injure et au déshonneur (dans la formulation "pré-réforme » de la provocation). Ce discours mobilise, ainsi que l'écrit Hart dans Punishment and Responsibility, "des inférences de sens commun sur la nature humaine », et fait partie intégrante des conceptions juridiques, mais aussi des normes culturelles et sociales sur la passion. Le même Hart, dans une remarque teintée d'ironie, ajoute ensuite que, parmi ces inférences se trouve le fait que «les hommes sont capables de se maîtriser devant un tiroir-caisse ouvert, mais pas lorsqu'ils sont mis face à une épouse adultère ${ }^{35}$. Reste à

33. Notons que le juge a un pouvoir très étendu au regard de la définition de l'excuse (en droits anglais et américain), puisque c'est lui qui contrôle la « recevabilité » de la défense, c'est-à-dire le fait que la question soit posée au jury, et que les instructions à ce dernier soient énoncées dans les termes de la provocation. Le jury est juge du fait, mais répond de manière binaire aux questions qui lui sont posées sur la culpabilité. Le fait qu'il puisse se prononcer sur ce point est important puisque, lorsque le jury délibère sur l'applicabilité de la défense aux faits, le juge peut prononcer ensuite un éventail de peines beaucoup plus large, d'une poignée d'années seulement à 15 ou 20 ans de prison (si le jury répond positivement) ou la réclusion à perpétuité (s'il répond négativement).

34. En réalité, le domaine des crimes dits passionnels, ou plutôt commis «sous l'empire de la passion », est un parfait exemple d'objet juridique faussement familier, que l'on a le sentiment de connaître alors même qu'on ne connaît pas le droit. D'une part, on se représente bien une sorte d'idéal-type de ce qui correspondrait en gros à un schéma de triangle amoureux à la fin tragique, domaine nourri d'un fort inconscient artistique et littéraire. D'autre part, c'est un objet juridique qui, malgré l'intérêt du législateur (dans les systèmes de common law, car la France a aboli le dispositif avec le Nouveau code pénal en 1994), n'a que très rarement fait l'objet d'études de synthèse, à l'inverse de ce qui s'est produit pour le crime de haine [hate crime], qui a notamment fait l'objet d'un " prototypage » dès la décennie 1990.

35. « As we consider these different cases, not only do we reach much vaguer concepts, but we become progressively more dependent on the agent's own statements about himself, buttressed by inferences from "common-sense" generalizations about human nature, such as that men are capable of self-control when confronted with an open till, but not when 
savoir de quoi sont faites ces généralisations, et à saisir les fondements du critère d'admission des émotions en droit pénal.

D'autre part, à ce discours très ancien, pierre de touche de la justification des "défenses émotionnelles», se superpose une structure des moyens de défense et un traitement des émotions par le droit peu compatibles, de fait, avec cette idée de concession générale à la faiblesse humaine. Tout d'abord, parce qu'une étude approfondie des textes et décisions montre que, sous cette référence mystérieuse et quelque peu lénifiante à la «nature humaine», et à des situations qui seraient « naturellement pousse-au-crime » pour ainsi dire, le droit des " défenses » opère en fait très souvent par une approche différenciée des émotions. En d'autres termes, on ressent une première perplexité devant un «tri pénal des émotions » que ne semble pas pouvoir justifier un consensus sur leur nature ou leurs manifestations typiques respectives, ou un accord sur une échelle commune de dangerosité associée aux affects ${ }^{36}$. Pourquoi, en effet, devraiton réserver un traitement plus favorable à la colère et au «feu de la passion », via l'admission de l'excuse de provocation dans certains cas d'homicide, quand il est impossible d'invoquer la compassion pour justifier ou excuser le même résultat, dans les systèmes où l'euthanasie active est fermement prohibée par la loi $?^{37}$ Comment séparer par conséquent le bon grain de l'ivraie des émotions, afin de justifier ce qui n'apparaît autrement que comme un privilège suspect? Deuxièmement, une excuse telle que la provocation ou le loss of control (dans un lexique plus contemporain) a pu faire l'objet d'une approche catégorielle très marquée, résultant du fait qu'elle n'est, à l'origine, qu'une transposition directe des lois de l'honneur dans le droit de l'homicide. La défense de provocation ne fut au départ disponible que pour des agents bien spécifiques, et placés dans une situation sociale particulière : combattants en duel, époux trompés surprenant leur femme infidèle - elle restait en revanche interdite aux fiancés trahis par leurs fiancées ou aux femmes trompées par leur mari, c'est pourquoi on a longtemps dit que la provocation était historiquement un moyen de sanction indirecte de l'épouse infidèle.

confronted with a wife in adultery », H. L. A. Hart, Punishment and Responsibility, Oxford University Press, 1968, p. 33.

36. La réputation de dangerosité supérieure et par conséquent la défiance associée aux émotions sthéniques relève d'une opposition contestable entre affects « actifs » (sthéniques), n'entravant pas mais exprimant les pouvoirs d'agir authentiques de l'agent, qui sont perçus par le droit avec une indulgence très limitée, et les affects passifs (dits asthéniques), vus comme paralysant l'action. Ces classifications incluent sous la première catégorie la colère, et sous la seconde la peur, plus facilement tolérée dans ses effets parce qu'elle aurait un effet «pré-incapacitant». On valoriserait donc dans la distribution des excuses les émotions entravant la maitrise de soi, tout en restant passives. Beaucoup d'auteurs, des positivistes italiens à Bentham, ont pourtant considéré que les auteurs d'homicides «provoqués » n'étaient pas des cibles convenables pour la dissuasion ou l'incapacitation.

37. Par exception, le suicide assisté est autorisé dans quelques États de l'Ouest américain : Oregon, Washington, Montana, Vermont, Californie (depuis 2015), Colorado (2016). 
En somme, dans cette première version - prémoderne - de la défense, il $\mathrm{y}$ a justement l'idée, dont personne ne songe à se cacher à l'origine, que toutes les émotions ne se valent pas socialement. Cette première approche en droit positif, ouvertement catégorielle, est progressivement abandonnée au fil des réformes, au profit d'une formulation se voulant moralement neutre - et, dans le cas du droit de la provocation, moins (ostensiblement) sexiste. Il s'agit en fait du Coroners and Justice Act de 2009 en droit anglais, qui tend notamment à évacuer la référence aux affects de type " sthénique », c'est-à-dire actifs - ainsi, les termes « rage », " passion », ne figurent plus désormais dans les dispositions correspondantes. Le législateur contemporain anglais ou américain y substitue le vocabulaire neutre ou prétendument neutre - de la «perte du contrôle de soi » [loss of selfcontrol] et de la "perturbation émotionnelle extrême" [extreme emotional disturbance], opérant un lissage visible des termes moraux généralement considéré comme lié à la conception libérale de la défense ${ }^{38}$. Des études relativement récentes sur la provocation en droit américain ${ }^{39}$ ont cependant permis de souligner que, non seulement l'approche catégorielle avait parfois survécu en pratique dans les États ayant adopté la nouvelle formulation - du fait de la fidélité marquée par le juge américain, dans son interprétation des dispositions nouvelles, à l'esprit de l'ancienne défense de common law, mais encore parce que cette approche différenciée (par catégories de justiciables) se trouvait même aggravée par l'élargissement du champ d'application de la défense moderne. En particulier, les liens d'intimité entre la victime et l'auteur n'étant plus limités légalement au strict lien matrimonial (comme c'était le cas antérieurement, dans la conception victorienne de la provocation), ce moyen de défense devenait accessible dans un cadre relationnel plus flou et indéterminé, ce qui a parfois donné lieu à des dérives. Celle-ci a pu être admise par le juge dans des cas semblant très éloignés de la figure de « vengeance du mari trompé » qui constituait la matrice historique de la défense : on pense ainsi aux cas limites évoqués par V. Nourse, tel celui de l'homme ayant voulu réparer l'outrage des moqueries d'une prostituée qu'il fréquentait, de tel auteur outragé par le refus par la victime de rapports intimes avec lui, ou d'un autre s'étant vengé de l'indifférence d'une femme ne désirant plus le voir, avant même d'avoir entamé avec lui une relation véritable $e^{40}$. De manière générale, on renverra pour une critique

38. Malgré le mouvement de reformulation, la défense «traditionnelle » de provocation, ou défense " de common law », demeure très puissante à la fois dans la pratique du droit et dans l'imaginaire collectif. En droit anglais, on voit par exemple que les juges sont réticents à appliquer des dispositions qui interdisent de considérer l'infidélité comme le seul élément déclencheur valide de l'acte provoqué, et les appliquent par conséquent de manière assez sélective. Voir sur ce point $R$. v. Clinton, [2012] EWCA Crim 2 ; $\$ 39-40$ et $\S 49$.

39. Victoria Nourse, «Passion's Progress: Modern Law Reform and the Provocation Defense ", in The Yale Law Journal, Vol. 106, $\mathrm{n}^{\circ} 5$ (mars 1997), p. 1331-1448. Sur le droit anglais, voir Jeremy Horder, Provocation and Responsibility, Clarendon Press, "Oxford Monographs on Criminal Law and Justice », 1992.

40. Voir Bedder v. Director of Public Prosecutions, 2 All E.R. 801 (H. L.1954) ; People v. Fardan, 628 N.E. 2d 41, 42 (N.Y. 1993). 
de fond de la défense historique de provocation à la théorie féministe, qui a minutieusement mis au jour l'implicite sexiste de la défense, y compris dans sa version "moderne», sous couvert même du recours à une neutralité renforcée des formules légales utilisées ${ }^{41}$.

\section{2) Approche évaluative et intelligibilité de l'excuse}

Une fois ces éléments de contexte posés, nous tenterons à présent de mettre en tension l'approche traditionnelle de l'excuse émotionnelle dans la philosophie pénale et les distinctions réelles à l'œuvre dans la jurisprudence et la doctrine angloaméricaine. L'approche dominante des excuses en théorie pénale - à laquelle on se référera ci-après sous le nom de «théorie orthodoxe de l'excuse " - s'identifie en effet peu ou prou à l'approche hartienne de l'excuse, c'est-à-dire une approche volontariste réduisant l'atténuation de culpabilité à une diminution de capacité. Cette réduction a notamment eu pour effet adjacent d'hypothéquer toute analyse sérieuse de la régulation des passions dans et par le droit pénal, en renvoyant l'excuse au domaine de la non-action. Hart donne notamment cette définition fameusement "négative» de la mens rea (intention coupable, l'un des éléments essentiels de l'infraction pénale) comme absence de condition d'excuse, l'excuse reposant à son tour sur l'absence de capacity ou de fair opportunity d'agir autrement ${ }^{42}$. Or, dans ce cadre traditionnel de compréhension de l'émotion - que l'on qualifiera, avec M. Nussbaum et D. M. Kahan, de «mécaniciste » - seule «la quantité ou l'intensité de l'émotion fournit une excuse, et non pas les raisons pesant en faveur de l'émotion ${ }^{43}$. Dans le temps précédent de l'analyse, nous avons soutenu une vision «non paresseuse » de l'excuse émotionnelle, c'est-à-dire pourvoyeuse d'intelligibilité et rationnelle. Il s'avère cependant que le maintien de cette exigence d'agentivité dans certains types d'excuse, et en particulier dans l'excuse émotionnelle, nécessite d'adopter une toute autre conception de l'émotion, en envisageant notamment qu'elle puisse représenter en ellemême une évaluation appropriée (du moins en partie) des circonstances de l'action. Par exemple, s'agissant de la contrainte :

41. Voir, pour une proposition d'abolition de la défense sur ce fondement, J. Horder, Provocation and Responsibility, Clarendon Press, 1992, p. 186-187.

42. H. L. A. Hart, Punishment and Responsibility, Oxford University Press, 1968, p. 152.

43. La citation prise dans son contexte est la suivante : «Under conventional liberal theory, [...] the quantity or intensity of the emotion provides the excuse, not the reasons for the emotion. This focus on emotion, to the exclusion of reason, reflects a very important assumption made by liberal theories of the defense, that emotion obscures reason. When we distinguish the rapist killer from the departing wife killer, we acknowledge a very different view of emotion, one in which emotion is imbued with meaning. Both the departing wife killer and the rapist killer may be upset, but the meanings embodied in their claims for emotional understanding are quite different. In distinguishing these cases based on the reasons for the claimed emotion, we acknowledge a view of emotion in which emotion is not the enemy of reason but, instead, its embodiment », V. Nourse, op. cit., p. 1389-1390. 
According to the evaluative view, duress exculpates not because (and when) threats vitiate a person's moral agency, but because (and when) a person's fear expresses a rational and morally appropriate assessment of her circumstances $^{44}$.

La nécessité d'opter pour une théorie évaluative - et non mécaniciste de l'émotion se fait d'autant plus pressante face au droit de la provocation, venu codifier en grande partie la référence de la culture populaire aux soidisant « crimes passionnels ». L'émotion ne devrait donc plus être analysée comme pure déviance quantifiable, comme franchissement d'un seuil qui ferait obstacle au cours d'agentivité ordinaire. Comme l'a souligné un auteur au sujet de la violence post-attentats du 11 septembre aux États-Unis, analysée comme une série de crimes of passion:

Crimes of passion stand as a particularly frank acknowledgment that emotion is not merely a deviance that can be regulated through the exercise of reason, but a constitutive part of human experience that sometimes gains the protection of law... Put differently, passion has honorary reason status ${ }^{45}$.

Ce «statut honoraire de raison» de la passion, telle qu'appréhendée par le droit, n'est le plus souvent pas apparent, et le cède la plupart du temps aux catégories de la perte de contrôle, toujours étroitement associées au droit pénal moderne.

Dans un article qui a fait date ${ }^{46}$, Martha C. Nussbaum et Dan M. Kahan démontrent les vertus descriptives d'une telle théorie évaluative de l'émotion en droit pénal. Cette conception se trouve par ailleurs pleinement en accord avec les avancées de la psychologie contemporaine, pour laquelle l'émotion - ou du moins, certaines émotions, telles que la colère et la peur - est constituée d'une évaluation par l'agent de sa situation. En d'autres termes, l'émotion est toujours mêlée d'un élément cognitif. Par suite, il est possible d'évaluer cet élément cognitif à la racine de l'émotion comme porteur d'un jugement - exact ou non - sur la situation de l'agent, et, partant, d'évaluer cette émotion comme « appropriée » ou non. L'émotion, «matière possible d'une culture de soi ${ }^{47}$, fait dans cette vision partie intégrante de la personnalité de l'agent. À partir de là, on comprend que l'usage d'une conception évaluative de l'émotion dans le champ du droit pénal modifie le sens de la référence à l'émotion dans l'excuse. Celle-ci atténue la peine, non parce qu'elle induirait une diminution de capacité (vision orthodoxe

44. Dan M. Kahan \& Martha C. Nussbaum, « Two Conceptions of Emotion in the Criminal Law », in Columbia Law Review, vol. 96, n² 2, 1996, p. 336.

45. Muneer Ahmad, «A Rage Shared by Law: Post-September 11 Racial Violence as Crimes of Passion », in California Law Review, vol. 92, n 5 (Oct. 2004), p. 1303.

46. Dan M. Kahan \& Martha C. Nussbaum, op. cit., p. 269-374.

47. Dan M. Kahan \& Martha C. Nussbaum, op. cit., p. 271-272. 
de l'excuse), mais parce que cette émotion exprime une évaluation par l'agent de sa propre situation jugée, en l'espèce, appropriée ${ }^{48}$. Autrement dit, l'émotion serait véritablement « validée » par le droit en ce qu'elle reposerait sur des états cognitifs jugés correspondre (moralement et/ou socialement) à la « réalité » de la situation de l'agent.

Or c'est peu de dire que l'immense majorité des doctrines du droit pénal sont insuffisantes sur ce plan, car elles ont presque toutes négligé à la fois l'influence de la conception évaluative de l'émotion sur la structure du droit substantiel et le pouvoir descriptif d'une telle conception ${ }^{49}$. Si l'on prend l'exemple du droit américain et de la doctrine du voluntary manslaughter («homicide volontaire »), dont on ne trouvera pas d'équivalent en droit français, il n'est pas possible de saisir ce qu'est un élément déclencheur adéquat [qualifying trigger] de la perte de contrôle chez l'agent sans adopter dans le même temps une approche évaluative. Par l'exigence d'une " provocation adéquate » en effet, qui décrit le premier temps de la double causalité vue plus haut, le droit ne se contente pas de demander la preuve qu'une volonté a été affaiblie, ou qu'une certaine passion était trop intense pour que l'agent puisse lui opposer sa résistance - dans cette hypothèse, un individu d'ordinaire très intempérant et colérique pourrait dès lors se prévaloir de l'excuse plus facilement qu'un agent tempérant. La notion de " provocation adéquate », cause éligible de la perte de contrôle, renvoie en réalité à une qualité de l'émotion, davantage qu'à son intensités0. On en donnera deux exemples récents, concernant des affaires dans lesquelles la provocation a été invoquée : dans le premier cas, elle a été admise, le second renvoyant à un cas générique où elle tendrait plutôt à être refusée. Le premier cas est celui d'une accusée qui tue un homme après avoir découvert qu'il avait violé sa fille, et après qu'il lui avait affirmé qu'il le referait ${ }^{51}$. Dans une perspective évaluative, on peut juger raisonnable sa motivation par l'émotion - la colère et la peur que l'agresseur ne recommence - tout en considérant que l'action reste injustifiée ou imparfaite: il y a d'autres manières, en effet, de régler ce problème pour le justiciable. Le second cas est celui de l'excuse dite de "panique homosexuelle» [gay panic defense]. Dans plusieurs affaires d'homicide, en effet, à compter des années cinquante $^{52}$, des accusés ont pu plaider - parfois avec succès - un moyen de

48. Dan M. Kahan \& Martha C. Nussbaum, op. cit., p. 305-306.

49. Conception que les auteurs font remonter à l'aristotélisme et au stoïcisme.

50. V. Nourse écrit, dans le même sens, que ce que l'on fait lorsqu'on distingue les causes éligibles et les causes non éligibles est déjà d'appliquer une théorie évaluative, en cela incompatible avec les prémisses libérales de l'excuse : en réalité toute perte de self-control « ne mérite pas la compassion du droit », V. Nourse, op. cit., p. 1333.

51. People v. Shields, 575 N.E. 2d 538, 546 (Ill. 1991) : la victime est un homme ayant admis qu'il avait violé la fille de l'auteur des faits et ayant affirmé qu'il le referait, non sans avoir également agressé physiquement l'accusée. Les circonstances furent jugées comme constituant une provocation suffisante.

52. Voir le procès inaugural de Charles Lawrence et Lewis Killen, en 1954, qui donne lieu à un verdict de culpabilité sur des charges de voluntary manslaughter, donc pour une 
défense partiel consistant à affirmer que l'homicide volontaire perpétré l'avait été en raison d'avances homosexuelles faites à l'auteur par la victime $^{53}$, dans un contexte socioculturel non exempt de tensions, et dans lequel l'American Psychiatric Association définissait l'homosexualité comme une maladie mentale. Bien que cette défense ait parfois pu être acceptée (elle a par exemple donné lieu à une condamnation particulièrement clémente à une peine de six ans d'emprisonnement par le juge Young dans le cas Thacker ${ }^{54}$, beaucoup de cours en refusent désormais l'accès, et celle-ci se voit désavouée tant au niveau de la législature d'État ${ }^{55}$ que par les juges ${ }^{56}$

peine réduite - mais maximale, dans cette catégorie - de vingt années de prison: "Charles Lawrence and Lewis Killen's trial for first-degree murder lasted three days and was turned over to the jury for deliberations on Saturday, November 6, at 10:30 p.m. Just four hours later the jury returned with a reduced verdict of manslaughter for both defendants. The principal reasons for the lesser conviction, according to one of the jurors, were Simpson's homosexuality and the state's failure to prove premeditation. Later that week the presiding judge, Grady Crawford, sentenced Lawrence and Killen to the maximum penalty, 20 years ». Sur cette affaire, on pourra consulter l'article en ligne d'Antolin Garcia Carbonell à l'adresse : http://biscaynetimes.com/index.php?option $=$ com_content\&view $=$ article\&id $=1521$ :homo -hysteria\&catid=46:features.

53. Voir par exemple le cas Thacker (cf. n. 58) et la trans panic defense dans la récente affaire Dixon (2013), où l'auteur, après avoir plaidé coupable d'homicide volontaire (son équivalent dans l'État de New York, le «manslaughter in the first degree », qui couvre notamment les heat of passion killings, les homicides par provocation), voit une peine de 12 ans prononcée à son encontre (pour une peine encourue de $25 \mathrm{ans}$ ), provoquant des réactions très vives chez les proches de la victime et les associations de défense des personnes transgenres : https://www.theguardian.com/us-news/2018/may/12/gay-panic-defence-tactic-ban-

court. Un cas intéressant et fort connu, enfin, celui de l'affaire Schmitz (People v. Schmitz, 586 N.W.2d 766, Michigan Court of Appeals 10 December 1998), suspend l'octroi de la défense à un bref délai de survenance de l'acte criminel à compter de la provocation : puisque Schmitz n'avait pas « réagi » à la provocation (une connaissance avait avoué son attirance pour lui en direct sur le plateau d'une émission de télévision) dans les trois jours, sa réponse ne pouvait être «immédiate» et il se vit ainsi condamné à une peine de 25 à 50 ans de réclusion criminelle pour meurtre. Une libération conditionnelle lui a été accordée en 2017.

54. Il s'agit d'une affaire moins connue que les cas de gay panic defense souvent mis en avant, jugée dans l'Utah en 1994, mais qui a donné lieu à des protestations très véhémentes de la part de groupes de défense L.G.B.T.I. Voir sur ce point la presse de l'époque : https://www.nytimes.com/1994/08/17/us/judge-draws-protests-after-cutting-sentenceof-gayman-s-killer.html ; ou encore : https://www.deseretnews.com/article/369999/JUDGEREDUCES-PENALTY-INPARKWEST-SLAYING.html.

55. La Californie a rendu illégale la possibilité d'une telle défense en 2014, et l'Illinois a suivi ses traces en 2018. Voir à nouveau : https://www.theguardian.com/us-news/2018/may/12/gaypanic-defence-tactic-ban-court. Enfin, sur l'exemple australien, on pourra se reporter à : https://www.theguardian.com/world/2017/mar/22/gay-panic-defence-thrown-out-inqueensland.

56. Cf. par exemple, au sujet du meurtre par un spectateur de deux femmes homosexuelles surprises en plein acte sexuel, en 1988 : Commonwealth v. Carr, 398 Pa. Superior Ct. 306 (1990), 580 A.2d 1362. La cour refuse fermement de reconnaître une "provocation adéquate » dans cette vision : « Whatever a person's views about homosexuality, the law does not condone or excuse the killing of homosexuals any more than it condones the killing of heterosexuals. Similarly, it does not recognize homosexual activity between two persons as legal provocation sufficient to reduce an unlawful killing of one or both of the actors by a third person from murder to voluntary manslaughter », p. 311 . 
et par l'association du barreau américain ${ }^{57}$. Quelle que soit l'intensité de l'émotion en cause, en effet, les juges ne s'occupent pas (ou pas seulement) de savoir si la provocation alléguée était suffisante ou pas, subjectivement, pour miner la maîtrise de soi de l'agent: au contraire, ils estiment la provocation insuffisante précisément parce qu'ils concluent que le droit devrait combattre ou du moins dénoncer l'évaluation de la situation faite par l'auteur. D'où le traitement différent réservé à la "mère justicière » et à la défense "de panique homosexuelle »: «[...] to lodge a defence that turns solely on the victim's sexual orientation or gender identity is really just using the bias of jurors and perhaps judges to their advantage and it perpetuates anti-LGBT stigma [...] and it's something that in 2018 we shouldn't permit anymore $»^{58}$. La colère, dans le premier cas, paraît au contraire "appropriée », ce qui la distingue juridiquement du second cas générique. La jurisprudence mentionne en outre souvent le fait que l'émotion est appropriée en matière de provocation car elle est réputée "moins haineuse »- ce qui pose d'ailleurs un problème conceptuel pour les crimes homophobes, classés parmi les crimes de haine mais bénéficiant encore (rarement à une époque récente, il est vrai) d'une excuse de provocation.

Pour conclure sur ces interprétations évaluatives de la défense de provocation, notons que, selon certains auteurs, la Law Commission anglaise tendrait malgré la formulation moderne de la provocation à y voir une «justification imparfaite» au lieu d'une «excuse compassionnelle» ${ }^{59}$. Autrement dit, il y aurait dans cet organe de proposition de réforme une tendance à intensifier les aspects «justificatoires» et rationnels de la défense. L'observation du droit américain est en revanche plus contrastée : si la nouvelle version de la défense dans le Model penal code n'implique pas textuellement de défaut de rationalité ${ }^{60}$, elle a parfois, du fait de l'usage des cours, été résolument poussée dans un sens mécaniciste, comprise comme formulant un degré de déficience de la volonté se plaçant juste au-dessus de l'insanity (abolition du discernement) ${ }^{61}$.

57. Celui-ci a adopté une résolution en 2013 contre l'usage de ce type de moyens de défense.

58. Cf. https://www.theguardian.com/us-news/2018/may/12/gay-panic-defence-tactic-ban-court.

59. «In sum, if the moral mark of the new Law Commission approach is that conduct is imperfectly rightful, and therefore both condemned and partially vindicated, the mark of the old law was that conduct was partially excused, both wrongful and partially condoned on ground of compassion. [...] It is (arguably) one thing to act out of morally appropriate anger, remaining in control of one's actions, the new approach. It can never be right at any level to lose one's control, for this entails a defect in one's rationality, the sine qua non of moral action. Loss of control, hijacking reason, is a problem from the start», Alan Norrie, "The Coroners and Justice Act 2009 - Partial Defences to Murder 1. Loss of Control », in Criminal Law Review, Vol. 275, 2010, p. 277 (nous soulignons). Voilà qui délimite, selon Norrie, deux « territoires » bien distincts pour l'ancienne et la nouvelle défense.

60. Rappelons qu'un meurtre devient un homicide volontaire «when it is committed under the influence of extreme mental or emotional disturbance for which there is a reasonable explanation or excuse », MPC, § 210.3.(1)(b), 1980 (nous soulignons).

61. On peut noter en effet l'extrême adaptabilité des dispositions de la reformulation du MPC, du fait de l'abandon de l'approche catégorielle présente dans la common law. Autrement dit, 
Bien que l'évaluation de l'émotion ne soit pas pour le droit pénal un objet ultime, puisqu'il se porte avant tout sur des actes, l'étude de ces excuses émotionnelles montre bien que l'évaluation de l'émotion participe de manière significative à l'évaluation de l'action. En matière d'excuses, la théorie de l'agentivité rendrait ainsi visible le filtrage de l'émotion par un critère d'intelligibilité de la motivation des agents. D'aucuns, dans la lignée des Critical Legal Studies, y voient l'indice de la présence d'un «voile» jeté par les catégories formelles du droit pénal moderne (comme l'est par exemple la notion vide de «self-control ») sur les fondements véritables de l'excuse émotionnelle, qui seraient évaluatifs et (moralement) substantiels, en dépit de l'apparent lissage du discours exclusivement privatif sur l'émotion ${ }^{62}$.

Le bénéfice de la "justification imparfaite » par l'émotion sanctionne ainsi positivement le fait d'avoir agi sur des motivations largement intelligibles, c'est-à-dire appropriées. Nous conclurons en prolongeant la réflexion par une illustration des effets de cette analyse qualitative de l'émotion - «raisonnable » en tant qu'elle est "motivée », et qui n'opère pas réduction de l'auteur à une dépossession radicale de ce qui le définit comme agent.

\section{3) La visibilité nouvelle du contexte social et politique de l'excuse}

Nous prendrons un seul exemple des conséquences de ce qui a été énoncé : la théorie évaluative de l'émotion et le maintien de l'agentivité dans l'excuse permettent d'envisager beaucoup plus naturellement les excuses en relation avec leur contexte politique et social immédiat. La minutieuse analyse par Muneer Ahmad des cas de violence post-11 septembre aux États-Unis est particulièrement éclairante à ce sujet ${ }^{63}$. L'auteur étudie le phénomène criminel (notamment les atteintes aux personnes, avec plusieurs

toute expérience affective de perte de contrôle semble suffisante pour désactiver le contrôle de la volonté et des raisons. Dans plusieurs cas où la défense d'EED a été appliquée, les accès de rage ne semblent pas pouvoir être compris du tout (aucune évaluation de l'émotion ne figure ainsi dans le jugement, et pourtant l'atténuation leur est accordée). Voir par exemple : State v. Elliott, 411 A.2d 3, 8 (Conn. 1979) ; People v. Casassa, 404 N.E.2d 1310 (N.Y. 1980).

62. Cette opération de dissimulation fait l'objet d'une étude minutieuse dans toute l'œuvre de A. Norrie, par exemple dans Crime, Reason and History. A Critical Introduction to Criminal Law, Cambridge University Press, «Law in Context», $3^{\mathrm{e}}$ éd., 2014. Elle est également décrite, dans une tout autre approche, dans l'article précité de Dan M. Kahan et Martha C. Nussbaum, "Two Conceptions of Emotion in the Criminal Law », in Columbia Law Review, vol. 96, $\mathrm{n}^{\circ} 2$, 1996, p. 373-374: «This ambivalence is counterproductive. Our primary normative claim in this Article has been that the law would be better if, in all instances, it were expressly evaluative. [...] Most important of all, evaluative doctrines are superior to mechanistic ones because they are honest. We have shown that terms like "premeditation", "impulse", and "voluntariness" frequently obscure the substance of evaluative appraisals $[\ldots] »$.

63. Muneer Ahmad, «A Rage Shared by Law : Post-September 11 Racial Violence as Crimes of Passion », in California Law Review, Vol. 92, n 5 (Oct., 2004), p. 1259-1330. 
cas d'homicides) en posant la question de l'attitude du public et du traitement judiciaire face aux meurtres de résidents américains et d'étrangers dans les mois qui ont suivi les attentats. La thèse de l'article est fondée sur une mise en regard de deux paradigmes : le crime de haine [hate crime] et le crime de passion [crime of passion]. Rappelant les trois caractéristiques du crime de haine - un biais ou préjugé personnel de l'auteur envers un groupe, une motivation perçue comme radicalement déviante et irrationnelle, un acte symbolique, visant exclusivement à communiquer sa haine du groupe - l'auteur explique pourquoi ces meurtres et agressions n'ont pas été vécus réellement comme des crimes de haines, alors même que certaines caractéristiques - un auteur et une victime inconnus l'un de l'autre, un acte criminel dirigé contre ce que représente la victime en tant que membre d'un groupe donné, le groupe flou visé étant ici celui des « personnes d'apparence musulmane » selon les auteurs - auraient pu les raccrocher à cette dernière catégorie. Or l'auteur explique pourquoi il n'en est rien, et le fait en plusieurs temps : 1) tout d'abord, c'est en réalité la passion, en tant que trope juridique et culturel, qui informe les déclarations et stratégies de défense de ces accusés, qui mettent en avant leur "patriotisme », leur honneur outragé, le besoin de "vengeance » irrépressible suite aux attentats. Selon une association originale mais efficace, l'auteur leur applique le réseau conceptuel du crime de passion : la patrie comme épouse violée et trahie par le terroriste, l'honneur national sali et qu'il faut laver dans le sang, la déloyauté des étrangers restant sur le sol américain, la violation de la «propriété » ultime qu'est la nation par un « intrus » ${ }^{64}$, etc. 2) Ensuite, c'est la distinction entre crimes de haine et crimes de passion qui va permettre de faire le lien avec le contexte socio-politique du crime. En effet, l'assimilation de cette série de crimes aux hate crimes, malgré l'aspect raciste et « gratuit» de ces actes, ne serait qu'une fausse caractérisation. La construction d'un acte comme hate crime permet en effet à l'observateur (et au juge) de se distancier le plus possible de l'auteur (comme monstrueux) et du crime (comme aberrant). L'acte est alors marqué comme clairement et intégralement aberrant, en vertu d'une déviance motivationnelle du perpétrateur dont l'observateur resterait exempté, pur, et à 1 'abri ${ }^{65}$. Cette distanciation maximale exclut évidemment toute cause d'excuse possible pour l'accusé. Enfin, l'explication véritable du crime par ses raisons n'a, dans ce contexte, pas réellement lieu, tant sa motivation nous demeure étrange - au sens fort du terme : extraneus, c'est-à-dire " en dehors de la rationalité ».

64. Cela dit beaucoup, souligne à raison l'auteur, de la psychologie nationale post-attentats, et notamment de la vision du rapport du citoyen à sa patrie, conçue comme "propriété » privée $\mathrm{du} \ll$ bon » citoyen.

65. « Hate crime is often viewed as an extreme or isolated phenomenon that involves conduct that is dramatic and aberrant and is perpetrated by deviant, rage-filled individuals who are "out of touch" with the rest of society " (Lu-In Wang, " "Suitable Targets" ? Parallels and Connections Between "Hate" Crimes and "Driving while Black" ", in Michigan Journal of Race and Law, vol. 6, n 2, 2001, p. 209-236 ; p. 211-212). 
À l'inverse, cet effet de distanciation morale est réduit à néant s'agissant de la motivation du crime de passion. Se penchant de très près sur la violence post-attentats (notamment aux États-Unis), Muneer Ahmad fait le constat que l'action dans ce cas n'est précisément pas perçue comme déviante et illogique de part en part. Bien que ces actes, et notamment les homicides et tentatives d'homicides, fassent l'objet d'une condamnation par la justice ${ }^{66}$, ils reposent dans leur motivation sur des croyances largement partagées, du moins pour la période allant de 2001 à (au moins) 2003 et la seconde guerre d'Irak couverte par l'étude. M. Ahmad conduit une analyse $\mathrm{du}$ traitement de ces affaires dans un contexte législatif, social et politique plus large. Selon lui, les motivations présidant à cette violence ont été quasinormalisées par les lois migratoires très fermes et définies par des biais ethniques $^{67}$, le profilage d'État, et le discours national sur l'immigration à l'époque. L'optique de vengeance de la majorité des auteurs a d'ailleurs pu trouver un écho dans le sens de la rétribution de l'Etat au travers du thème de la "guerre contre le terrorisme ». On comprend ainsi l'association aux crimes de passion et la différence de taille avec l'idéal-type du crime de haine : la distinction entre les premiers et les seconds passe par la distance morale que peut mettre le public - ou l'institution - entre lui-même et l'auteur des crimes. D'une manière générale, il existe une corrélation directe entre le "degré de rationalité » accordé à un préjugé ou une croyance déterminante chez un agent d'une part, et le degré de condamnation (sociale, morale, institutionnelle) et le traitement institutionnel de ce crime, d'autre part. Tout justiciable se prévalant d'un moyen de défense est alors traité, comme l'écrit V. Nourse, «exactement comme s'il était l'État», en ce qu'il représente à la fois « un risque politique et une possibilité [d'agir] pour la communauté », pour le public. C'est en ce sens que Nourse parle de nature «métonymique» de l'excuse en droit pénal : l'admission d'une défense est métonymique, car elle fait en quelque sorte du justiciable la partie pour le tout étatique. En somme, «le justiciable est représentant de nos peurs à propos de la gouvernance », écrit-elle encore ${ }^{68}$. Cette nature métonymique de l'excuse (que l'on retrouve dans le paradigme de la passion en droit) est on

66. L'un des auteurs, condamnés à mort pour le meurtre d'un homme sikh, a été exécuté en 2011 .

67. Soit la loi migratoire est neutre quant à l'ethnie et appliquée de manière biaisée, sélective (notification rapide après changement d'adresse par exemple : on note un "racially targeted use of the change of address requirement », y compris pour les résidents permanents), soit la loi fait ouvertement la différence entre les populations acceptées, en distinguant Arabes, musulmans, et personnes originaires du Sud-Est asiatique. Un exemple en est le «Special Registration Program » qui exige que les immigrants mâles venus d'une liste de 26 pays soient soumis à un interrogatoire (cela date de 2003). De même, concernant l'asile, au début de la guerre d'Irak, les immigrants venus de 33 pays énumérés sont sujets à la détention obligatoire.

68. V. Nourse, « Reconceptualizing Criminal Law Defenses », in University of Pennsylvania Law Review, vol. 151, $\mathrm{n}^{\circ} 5$ (mai 2003), p. 1691-1746 (nous traduisons) ; voir p. ex. p. 1706 : "The intellectual structure of this discourse, then, is metonymic; the defendant stands in for our fears about governance ». 
ne peut plus éloignée du crime de haine, puisque faire entrer un crime dans cette catégorie revient à localiser entièrement les causes du crime dans une idiosyncrasie aberrante, dans des émotions, préjugés et dispositions qui ne sont que (et ne peuvent être que) ceux de l'accusé.

On se souvient que, pour Hart, l'excuse accordée à l'agent est le sommet de l'individualisation : les excuses seraient même le prix individuel à payer pour avoir un système pénal dont la finalité est de protéger le pouvoir de choix ou la capacité des agents à s'autodéterminer, c'est-à-dire de protéger vraiment et effectivement l'autonomie de choix de l'individu. L'accent mis sur l'agent - en particulier dans les défenses - est donc en quelque sorte l'hommage que le système pénal rend à ses justiciables ${ }^{69}$.

Pour la théorie orthodoxe, les excuses sont ainsi la marque d'un droit pénal progressiste et moderne qui, mitigeant son égalitarisme strict et son principe de neutralité, consent à « individualiser » localement et par exception l'application de la loi. Or à la lumière de cette étude du rôle des émotions dans les excuses, il semble que le monopole d'interprétation de l'excuse par un consensus sur l'hyper-individualisation et l'exception doive être remise en cause. La catégorie d'excuse elle-même bénéficie d'une «promotion» au «statut honoraire de raison» qui va de pair avec la reconnaissance par le droit d'une lisibilité de l'émotion. On s'autorisera à terminer sur une question: à la suite de cet examen du rôle de l'émotion dans l'excuse, est-il bien certain que tout élément utile pour comprendre l'excuse se trouve réellement incorporé dans l'individu, qu'il réside exclusivement dans l'esprit ou le corps de cet agent? La réponse est, à ce qu'il semble, incluse dans la question.

69. H. L. A. Hart, 1968, op. cit., p. 136-137 (nous traduisons). 